\title{
PROBLEMS IN UNBOUNDED CYLINDRICAL DOMAINS
}

\author{
PATRICK GUIDOTTI \\ Mathematics Department, \\ University of California, \\ Patrick Guidotti, \\ 103 Multipurpose Science and Technology Bldg, \\ Irvine, CA 92697, \\ USA
}

\section{Introduction}

In this talk we present an approach to (Initial) Boundary Value Problems on unbounded domains of cylindrical type

$$
\mathbb{R}^{m} \times \Omega \text { for } \Omega \subset \mathbb{R}^{n} \text { open and bounded, } m, n \in \mathbb{N} \text {. }
$$

We combine the classical point of view of special functions with functional calculus for sectorial operators to obtain semi-explicit representation formulæ for the solutions. The formulæ are well suited for the further study of their properties as well as for the precise characterization of asymptotic behaviour in the large or for vanishing parameters (such as in perturbation theory). The approach is developed in [7] and extended in [6]. Here we briefly review its main ingredients and then consider a series of examples to illustrate the wide range of applicability of the ideas.

\section{Generalized Fundamental Solutions}

When analyzing Initial Boundary Value Problems (IBVPs) in cylindrical domains two general approaches are available. The classical approach based on finding a Green's function $G \in \mathcal{D}^{\prime}\left(\mathbb{R}^{m} \times \Omega \times \mathbb{R}^{m} \times \Omega\right)$ for the differential operator involved, or, in other words, the distributional kernel of the solution operator. If a Green's function exists, then solutiona are simply given by

$$
u(x)=\int_{\mathbb{R}^{m} \times \Omega} G(x, y) f(y) d y
$$


whenever the integral can be computed (possibly in the sense of distributions). The other approach rests on the theory of operator sums the main results of which are found in [9] and [4]. Their methods are functional analytical in nature but are based on different integral representations for the inverse of the sum.

We present a mixed approach which combines the classical approach with the functional analytical one which seems to be a very natural choice for the type of domain considered here. The basic idea is to treat the unbounded directions as in the classical theory and to condense the problem in the bounded part of the domain into an abstract operator. To implement this idea we shall need to introduce the concept of operator-valued fundamental solution which is a natural extension of the classical definition. In doing so we are able to integrate the explicit formulæ provided by the classical theory and the abstract operator formulations for BVPs in bounded domains.

\subsection{Some Definitions and Notations}

In this section we shall give a very brief overview of the main concepts and results concerning vector-valued distributions which are treated more extensively in [10] or [1].

As in the scalar case one defines the space of rapidly decreasing test functions by

$$
\left\{\varphi \in \mathrm{C}^{\infty}\left(\mathbb{R}^{m}, E\right)\left|\forall k, j \in \mathbb{N} \sup _{x \in \mathbb{R}^{m},|\alpha| \leq k}\left(1+|x|^{2}\right)^{j / 2}\right| \partial^{\alpha} \varphi(x) \mid \leq c_{k, j}<\infty\right\}
$$

where $E$ is a Banach space. For our purposes we shall choose $E$ to be either a space of functions or of linear and continuous operators between function spaces. The seminorms

$$
q_{k, j}(\varphi)=\sup _{x \in \mathbb{R}^{m},|\alpha| \leq k}\left|\partial^{\alpha} \varphi(x)\right|
$$

generate the standard locally convex topology of $\mathcal{S}\left(\mathbb{R}^{m}, E\right)$. If we denote by $\mathcal{S}^{\prime}\left(\mathbb{R}^{m}\right)=\mathcal{L}\left(\mathcal{S}\left(\mathbb{R}^{m}\right), \mathbb{K}\right)$ the space of scalar tempered distributions, then the corresponding space of vector-valued distributions is given by

$$
\mathcal{S}^{\prime}\left(\mathbb{R}^{m}, E\right)=\mathcal{L}\left(\mathcal{S}\left(\mathbb{R}^{m}\right), E\right) .
$$

Now, as in the scalar case, we have that

$$
\left[\varphi \mapsto \int_{\mathbb{R}^{m}} f(x) \varphi(x) d x\right]: \mathcal{S}\left(\mathbb{R}^{m}\right) \rightarrow E
$$


defines a tempered distribution for any locally integrable $E$-valued function. It follows that

$$
\mathrm{L}_{1, l o c}\left(\mathbb{R}^{m}, E\right) \hookrightarrow \mathcal{S}^{\prime}\left(\mathbb{R}^{m}, E\right),
$$

and any distribution which can be represented by a locally integrable function is called regular. The Fourier transform is given by

$$
\mathcal{F} \varphi=\hat{\varphi}=\frac{1}{(2 \pi)^{n / 2}} \int_{\mathbb{R}^{m}} e^{-i x \xi} \varphi(x) d x
$$

for any test function $\varphi \in \mathcal{S}\left(\mathbb{R}^{m}, E\right)$ and by

$$
\langle\mathcal{F} u, \varphi\rangle=\langle\hat{u}, \varphi\rangle=\langle u, \hat{\varphi}\rangle, \varphi \in \mathcal{S}\left(\mathbb{R}^{m}, E\right)
$$

for any tempered distribution $u \in \mathcal{S}^{\prime}\left(\mathbb{R}^{m}, E\right)$. It can be proven that

$$
\mathcal{F} \in \mathcal{G} \mathcal{L}\left(\mathcal{S}\left(\mathbb{R}^{m}, E\right)\right) \cap \mathcal{G} \mathcal{L}\left(\mathrm{L}_{2}\left(\mathbb{R}^{m}, E\right)\right) \cap \mathcal{G} \mathcal{L}\left(\mathcal{S}^{\prime}\left(\mathbb{R}^{m}, E\right)\right)
$$

In [3] vector-valued Besov spaces

$$
\mathcal{S}\left(\mathbb{R}^{m}, E\right) \hookrightarrow \mathrm{B}_{p, q}^{s}\left(\mathbb{R}^{m}, E\right) \hookrightarrow \mathcal{S}^{\prime}\left(\mathbb{R}^{m}, E\right)
$$

for $s \in \mathbb{R}$ and $p, q \in[1, \infty]$ are introduced via diadic resolutions of the identity in Fourier space. We refer the reader to the cited paper for the details. The scala of Besov spaces comprises several special function spaces. For instance, classical spaces of Hölder continuous functions

$$
\begin{aligned}
\operatorname{BUC}^{k+\alpha}\left(\mathbb{R}^{m}, E\right)= & \left\{u \in \operatorname{BUC}^{k}\left(\mathbb{R}^{m}, E\right) \mid\right. \\
& {\left.\left[\partial^{\alpha} u\right]_{\alpha}=\sup _{x \neq y} \frac{\left\|\partial^{\alpha} u(x)-\partial^{\alpha} u(y)\right\|_{E}}{|x-y|^{\alpha}}<\infty,|\alpha|=k\right\} }
\end{aligned}
$$

where $k \in \mathbb{N}$ and $\alpha \in(0,1)$, are obtained from the general scala setting $s=k+\alpha$ and $p=q=\infty$. The intrinsic norm

$$
\|\cdot\|_{\mathrm{BUC}^{k+\alpha}}=\sup _{|\alpha| \leq k}\left\|\partial^{\alpha} \cdot\right\|_{\infty}+\sup _{|\alpha|=k}\left[\partial^{\alpha} \cdot\right]
$$

is equivalent to the Besov norm. Other spaces which fall into the Besov category are for instance Sobolev-Slobodeskii spaces

$$
\begin{aligned}
\mathrm{W}_{p}^{s}\left(\mathbb{R}^{m}, E\right) & =\left\{u \in \mathrm{W}_{p}^{[s]}\left(\mathbb{R}^{m}, E\right) \mid\right. \\
{\left[\partial^{\alpha} u\right]_{s-[s]} } & \left.=\int_{\mathbb{R}^{m} \times \mathbb{R}^{m}} \frac{\left\|\partial^{\alpha} u(x)-\partial^{\alpha} u(y)\right\|_{E}^{p}}{|x-y|^{m+(s-[s]) p}} d(x, y)<\infty,|\alpha|=[s]\right\} .
\end{aligned}
$$


They correspond to choosing $s \in \mathbb{R}^{+} \backslash \mathbb{N}$ and $p=q \in(1, \infty)$ and their intrinsic integral norms

$$
\|\cdot\|_{\mathrm{W}_{p}^{s}}=\left(\sum_{|\alpha| \leq[s]}\left\|\partial^{\alpha} \cdot\right\|_{\mathrm{L}_{p}}^{p}+\sum_{|\alpha|=[s]}\left[\partial^{\alpha} \cdot\right]_{s-[s]}^{p}\right)^{1 / p}
$$

are equivalent to the Besov ones. We recall that $[s]=\max _{k \in \mathbb{N}}\{k \leq s\}$ and that $\mathrm{W}_{p}^{[s]}\left(\mathbb{R}^{m}, E\right)$ are the classical Sobolev spaces. In analyzing the mapping properties of generalized fundamental solutions below we shall at times make use of a Fourier multiplier theorem by Amann [3]. To formulate the theorem we need to introduce multiplier spaces first. Let $E$ and $F$ be Banach spaces and $\mathcal{L}(E, F)$ be the space of linear and continuous operators from $E$ into $F$. Define

$$
\begin{aligned}
& S^{k}\left(\mathbb{R}^{m}, \mathcal{L}(E, F)\right)=\left\{a \in \mathrm{C}^{m+1}\left(\mathbb{R}^{m} \backslash\{0\}, \mathcal{L}(E, F)\right) \mid\right. \\
& \left.\left\|\partial^{\alpha} a(x)\right\|_{\mathcal{L}(E, F)} \leq c(1+|x|)^{k-|\alpha|}, x \neq 0,|\alpha| \leq m+1\right\}
\end{aligned}
$$

for $k \in \mathbb{R}$.

Theorem 2.1. Let $k \in \mathbb{R}$. Then

$$
a(D)=\mathcal{F}^{-1} a \mathcal{F} \in \mathcal{L}\left(\mathrm{B}_{p, q}^{s+k}\left(\mathbb{R}^{m}, E\right), \mathrm{B}_{p, q}^{s}\left(\mathbb{R}^{m}, F\right)\right)
$$

for $s \in \mathbb{R}$ and $p, q \in[1, \infty]$ whenever $a \in S^{k}\left(\mathbb{R}^{m}, \mathcal{L}(E, F)\right)$. Moreover $a(D)$ depends continuously on a.

This theorem is a special case of [Theorem 6.2][3]. We are now ready for the definition of generalized fundamental solutions.

Definition 2.1. Let $\mathrm{P}=\sum_{|\alpha|<k} p_{\alpha} \partial^{\alpha}$ be a differential operator on $\mathbb{R}^{m}$ with coefficients $a_{\alpha} \in \mathcal{L}(E, F)$. Then we call a distribution

$$
G \in \mathcal{S}^{\prime}\left(\mathbb{R}^{m}, \mathcal{L}(F, E)\right)
$$

a fundamental solution for $\mathrm{P}$ iff

$$
\mathrm{P} G=\mathbf{1}_{\mathcal{L}(E, E)} \delta_{x} .
$$

Now a convolution can be introduced for vector valued functions and distributions spaces based on continuous multiplication operators for the underlying image spaces. In our situation we choose the multiplication given by

$$
\mathcal{L}(E, F) \times E \rightarrow F,(A, x) \mapsto A x
$$


Then it is a consequence of [Theorem 3.1][3] that the convolution operator

$$
\mathcal{S}^{\prime}\left(\mathbb{R}^{m}, \mathcal{L}(E, F)\right) \times \mathcal{S}\left(\mathbb{R}^{m}, E\right) \rightarrow \mathrm{C}^{\infty}\left(\mathbb{R}^{m}, F\right),(u, \varphi) \mapsto u * \varphi
$$

is bilinear and hypocontinuous (which means continuous in both variables, uniformly with respect to each variable separately if the other is restricted to a bounded subset). If $u$ satisfies integrability properties then the convolution is realized as an integral

$$
(u * \varphi)(x)=\int_{\mathbb{R}^{m}} u(x-\tilde{x}) \varphi(\tilde{x}) d \tilde{x} .
$$

As in the scalar case, once the existence of a fundamental solution

$$
G \in \mathcal{S}^{\prime}\left(\mathbb{R}^{m}, \mathcal{L}(F, E)\right)
$$

has been established, one can produce solutions for

$$
\mathrm{P} u=f \in \mathcal{S}^{\prime}\left(\mathbb{R}^{m}, F\right)
$$

by convolution $u=G * f$, whenever the convolution makes sense. Furthermore, if the Fourier transform of the fundamental solution $\hat{G}$ can be computed, Theorem 2.1 allows us to analyze its mapping properties. In the next section we shall consider some particular situations in which the above anisotropic fundamental solution approach can be applied directly or indirectly. We shall give examples in the parabolic, elliptic and hyperbolic cases.

\section{Examples and Applications}

\subsection{Elliptic Regularizations of Parabolic Problems}

Consider the problem

$$
-\varepsilon u_{t t}+u_{t}+A u=\tilde{f}(t), t \in \mathbb{R}
$$

It is called elliptic regularization of the parabolic problem obtained by setting $\varepsilon=0$ and considering only $t>0$. For simplicity we assume that $-A$ is the $\mathrm{L}_{p}(\Omega)$-realization of the Laplacian on $\Omega$ with homogeneous Dirichlet boundary conditions, that is, with domain of definition $\operatorname{dom}(A)$ given by

$$
\mathrm{W}_{p, 0}^{2}(\Omega)=\mathrm{W}_{p}^{2}(\Omega) \cap \stackrel{0}{\mathrm{~W}}{ }_{p}^{1}(\Omega) .
$$

$A$ is therefore a positive sectorial operator.

Lemma 3.1.

$$
G_{\varepsilon}(t, A)=(1+4 \varepsilon A)^{-\frac{1}{2}} e^{\frac{t}{2 \varepsilon}-\frac{|t|}{2 \varepsilon}(1+4 \varepsilon A)^{\frac{1}{2}}}
$$

is a generalized fundamental solution for $-\varepsilon \partial_{t t}+\partial_{t}+A$. 
Proof. Consider the Fourier symbol

$$
a(\xi, A)=\varepsilon \xi^{2}-i \xi+A, \xi \in \mathbb{R}
$$

of $-\varepsilon \partial_{t t}+\partial_{t}+A$. Since $A$ is sectorial with angle larger than $\pi / 2$ Dunford calculus gives

$$
\begin{aligned}
& \left(-\varepsilon \partial_{t t}+\partial_{t}+A\right)^{-1}=\mathcal{F}^{-1}\left[\int_{\Gamma}\left(\varepsilon \xi^{2}-i \xi+\lambda\right)^{-1}(\lambda+A)^{-1} d \lambda\right] \mathcal{F} \\
& =\left[\int_{\Gamma} \frac{e^{\frac{t}{2 \varepsilon}-\frac{|t|}{2 \varepsilon}(1+4 \varepsilon \lambda)^{\frac{1}{2}}}}{(1+4 \varepsilon \lambda)^{\frac{1}{2}}}(\lambda+A)^{-1} d \lambda\right] *=(1+4 \varepsilon A)^{-\frac{1}{2}} e^{\frac{t}{2 \varepsilon}-\frac{|t|}{2 \varepsilon}(1+4 \varepsilon A)^{\frac{1}{2}}} *
\end{aligned}
$$

where ${ }^{*}$ indicates that the operator acts as a vector-valued convolution (in the variable $x$ ) with the specified kernel. We observe that the Fourier transform and the integral can be interchanged because the decay properties of the integrand and the continuous dependence of the functional calculus on the parameter $x$ which is proved in [Lemma 4.1.1][2]).

Denote the solution of (1) by $u^{\varepsilon}$. For $\tilde{f} \in \operatorname{BUC}^{\alpha}\left(\mathbb{R}, \mathrm{L}_{p}(\Omega)\right)$, for instance, it is given by

$$
u^{\varepsilon}(t)=\int_{-\infty}^{\infty} G_{\varepsilon}(t-\tau, A) \tilde{f}(\tau) d \tau .
$$

Theorem 3.1. Let $\tilde{f} \in \operatorname{BUC}^{\alpha}\left(\mathbb{R}, \mathrm{L}_{p}(\Omega)\right)$. Then

$$
u^{\varepsilon} \longrightarrow u(\varepsilon \rightarrow 0) \text { in } \mathrm{BUC}^{1+\alpha}\left(\mathbb{R}, \mathrm{L}_{p}(\Omega)\right) \cap \mathrm{BUC}^{\alpha}\left(\mathbb{R}, \mathrm{W}_{p, 0}^{2}(\Omega)\right)
$$

where u solves

$$
\begin{cases}u_{t}+A u & =\tilde{f}(t), t>0 \\ u(0) & =\int_{-\infty}^{0} e^{\tau A} \tilde{f}(\tau) d \tau\end{cases}
$$

Proof. Consider first the behavior of $G_{\varepsilon}(t, A)$ as $\varepsilon$ tends to 0 . If $t<0$, then

$$
G_{\varepsilon}(t, A)=(1+4 \varepsilon A)^{-\frac{1}{2}} e^{\frac{t}{2 \varepsilon}+\frac{t}{2 \varepsilon}(1+4 \varepsilon A)^{\frac{1}{2}}} \longrightarrow 0(\varepsilon \rightarrow 0) .
$$

On the other hand, when $t>0$,

$$
G_{\varepsilon}(t, A)=(1+4 \varepsilon A)^{-\frac{1}{2}} e^{\frac{t}{2 \varepsilon}-\frac{t}{2 \varepsilon}(1+4 \varepsilon A)^{\frac{1}{2}}} \longrightarrow e^{-t A}(\varepsilon \rightarrow 0)
$$

since

$$
\frac{1}{2 \varepsilon}-\frac{1}{2 \varepsilon}(1+4 \varepsilon A)^{\frac{1}{2}} \longrightarrow-A(\varepsilon \rightarrow 0) .
$$


In the limit, one therefore obtains

$$
\begin{aligned}
u^{\varepsilon}(t)=\int_{-\infty}^{\infty} G_{\varepsilon}(t-\tau, A) \tilde{f}(\tau) d \tau \longrightarrow \int_{-\infty}^{t} e^{-(t-\tau) A} \tilde{f}(\tau) d \tau \\
=e^{-t A} \int_{-\infty}^{0} e^{\tau A} \tilde{f}(\tau) d \tau+\int_{0}^{t} e^{-(t-\tau) A} \tilde{f}(\tau) d \tau
\end{aligned}
$$

which is the solution of the claimed parabolic problem. The fact that the convergence takes place in the asserted topologies follows from the analysis of the convergence of the symbols $a_{\varepsilon}^{-1}$ as $\varepsilon$ tends to zero, that is,

$$
\left(\varepsilon \xi^{2}-i \xi+A\right)^{-1} \longrightarrow(-i \xi+A)^{-1}
$$

in the symbol classes

$$
S^{-1}\left(\mathbb{R}, \mathcal{L}\left(\mathrm{L}_{p}(\Omega), \mathrm{L}_{p}(\Omega)\right)\right) \text { and } S^{0}\left(\mathbb{R}, \mathcal{L}\left(\mathrm{L}_{p}(\Omega), \mathrm{W}_{p, 0}^{2}(\Omega)\right)\right)
$$

and from Theorem 2.1.

It follows that, choosing

$$
\tilde{f}(t)=\left\{\begin{array}{ll}
f(t), & t>0 \\
A u_{0}, & t \leq 0
\end{array},\right.
$$

for $f \in \mathrm{BUC}^{\alpha}\left(\mathbb{R}^{+}, \mathrm{L}_{p}(\Omega)\right)$ and $u_{0} \in \mathrm{W}_{p, 0}^{2}(\Omega)$ one obtains the solution of

$$
\begin{cases}u_{t}+A u & =f(t), t>0 \\ u(0) & =u_{0}\end{cases}
$$

in the limit as $\varepsilon$ tends to 0 for the solution $u^{\varepsilon}$ of

$$
-\varepsilon u_{t t}+u_{t}+A u=\tilde{f}(t), t \in \mathbb{R} .
$$

In this way the compatibility condition

$$
A u_{0}=f(0)
$$

needed to obtain smoothness up to $t=0$ for the solution of the parabolic problem (see for instance [8]) appears to be related to the natural assumption

$$
\tilde{f} \in \mathrm{BUC}^{\alpha}\left(\mathbb{R}, \mathrm{L}_{p}(\Omega)\right)
$$

for the associated elliptic regularization. 


\subsection{Abstract Elliptic Problems}

Consider the abstract elliptic problem

$$
-\triangle u+A u=f \text { in } \mathbb{R}^{m}
$$

in $E_{0}$ where $A$ is a positive sectorial operator with domain of definition $E_{1}$. Problems of this type naturally arise from boundary value problems in cylindrical domains (see [7]) and fit into the framework of generalized fundamental solutions.

Either by taking a Fourier transform or, directly by analogy to the scalar case a fundamental solution can be found. The following theorem deals with dimensions 1,2 and 3. Analogous formulæ are valid in higher dimensions.

Theorem 3.2. A generalized fundamental solution can be found for $-\triangle+$ $A$ and is given by

$$
G_{m}(x, A)=\left\{\begin{array}{ll}
\frac{1}{2} A^{-\frac{1}{2}} e^{-|x| A^{\frac{1}{2}}}, & m=1, \\
K_{0}\left(|x| A^{\frac{1}{2}}\right), & m=2, \\
\frac{1}{|x|} e^{-|x| A^{\frac{1}{2}}}, & m=3 .
\end{array} \quad x \in \mathbb{R}^{m}\right.
$$

A proof of this result which is based on the Dunford functional calculus for sectorial operators can be found in [7]. It is interesting to observe that the fundamental solution can be understood in terms of an analytic function of the pseudodifferential operator $\sqrt{A}$. In odd dimensions, even in terms of the semigroup generated by $\sqrt{A}$. Based on Theorem 2.1 it is also easy to obtain associated regularity results in anisotropic spaces.

\section{Theorem 3.3.}

$$
\begin{aligned}
& {\left[f \mapsto G_{m}(\cdot, A) * f\right]} \\
& \in \mathcal{L}_{i s}\left(\mathrm{~B}_{q, r}^{t}\left(\mathbb{R}^{m}, E_{0}\right), \mathrm{B}_{q, r}^{t+2}\left(\mathbb{R}^{m}, E_{0}\right) \cap \mathrm{B}_{q, r}^{t}\left(\mathbb{R}^{m}, E_{1}\right)\right)
\end{aligned}
$$

Proof. We know that $\hat{G}_{m}(\cdot, A)=\left(\xi^{2}+A\right)^{-1}$. Recall that the operator $A$ is sectorial. In particular we have

$$
\left\|\left(\xi^{2}+A\right)^{-1}\right\|_{\mathcal{L}\left(E_{0}\right)} \leq c
$$

and

$$
\left\|\left(1+\xi^{2}\right)\left(\xi^{2}+A\right)^{-1}\right\|_{\mathcal{L}\left(E_{0}\right)} \leq c .
$$


Moreover

$$
\partial_{\xi}^{\alpha}\left(\xi^{2}+A\right)^{-1}=p_{\alpha}(\xi)\left(\xi^{2}+A\right)^{-|\alpha|-1}
$$

for any $\alpha \in \mathbb{N}^{m}$ and some polynomial $p_{\alpha}$ of order at most $|\alpha|$. Theorem 2.1 therefore applies and we obtain the desired regularity results.

To further illustrate the applicability of ideas we consider a fourth order boundary value problem in an infinite strip. The BVP reads

$$
\left\{\begin{aligned}
\triangle^{2} u & =f(x, y), & & \text { in } \mathbb{R} \times(0,1) \ni(x, y), \\
u & =\partial_{y} u=0, & & \text { on } \mathbb{R} \times\{0\} \\
u & =\partial_{y} u=0, & & \text { on } \mathbb{R} \times\{1\}
\end{aligned}\right.
$$

Again we can "pack" the part of the operator acting on the bounded direction into an abstract operator

$$
A=\partial_{y y y y}: \operatorname{dom}(A) \subset \mathrm{L}_{p}(0,1) \rightarrow \mathrm{L}_{p}(0,1)
$$

defined through

$$
\operatorname{dom}(A)=\left\{u \in \mathrm{W}_{p, \mathcal{B}}^{4}(0,1) \mid u(j)=u_{y}(j)=0, j=0,1\right\}
$$

and look for a solution satisfying the abstract (infinite dimensional) elliptic problem

$$
\left(\partial_{x x x x}-2 \sqrt{A} \partial_{x x}+A\right) u=f(x)
$$

for the vector valued function $u: \mathbb{R} \rightarrow \mathrm{L}_{p}(0,1)$ for $p \in(1, \infty)$. DunfordSchwartz operator calculus and Fourier transform allow us to explicitly compute the fundamental solution in this case, too.

Theorem 3.4. The abstract elliptic problem (7) possesses an operator valued fundamental solution $G \in \mathrm{L}_{1}\left(\mathbb{R}, \mathcal{L}\left(\mathrm{L}_{p}(0,1)\right)\right)$ given by

$$
G(x, A)=\frac{1}{2} A^{-1 / 2}\left(|x|+A^{-1 / 4}\right) e^{-|x| A^{1 / 4}}, x \in \mathbb{R}
$$

Proof. The fourth root of the sectorial operator $A$ can be defined by functional calculus and $G$ be interpreted by means of the semigroup generated by $-A^{1 / 4}$. A direct calculation then shows that it is indeed a fundamental solution. Alternatively the use of the Fourier transform allows us to relate 
the operator-valued case to the underlying scalar one. In fact

$$
\begin{aligned}
2 G(x, A) & =A^{-1 / 2}\left(|x|+A^{-1 / 4}\right) e^{-|x| A^{1 / 4}} \\
& =\int_{\Gamma} \lambda^{-1 / 2}\left(|x|+\lambda^{-1 / 4}\right) e^{-|x| \lambda^{1 / 4}}(\lambda-A)^{-1} d \lambda \\
& =\int_{\Gamma} \mathcal{F}^{-1}\left(\xi^{2}+\lambda\right)^{-2}(\lambda-A)^{-1} \mathcal{F} d \lambda=\mathcal{F}^{-1}\left(\xi^{2}+A\right)^{-2} \mathcal{F}
\end{aligned}
$$

where the first integral converges since $A$ is an invertible sectorial operator and the scalar factor is exponentially decaying along any path of integration avoiding (enclosing) the spectrum of $A$. The second equality is valid since the Fourier transform is continuous, for instance on $\mathrm{L}_{1}\left(\mathbb{R}, \mathrm{L}_{p}(0,1)\right)$, and because of the smooth dependence of the functional calculus on the variable $x \in \mathbb{R}$ (cf. [Lemma 4.1.1][2]). In the last equality we made use of the fact that the Fourier transform of the scalar factor in the integrand is know, see for instance [Chapter 17][5].

The solution can therefore be understood in terms of semigroups generated by first order pseudodifferential operators and their resolvents. This shows how "the different directions" contribute to the full solution operator. Using theorem 2.1 it is possible to characterize the anisotropic mapping properties of the associated convolution operator.

Theorem 3.5. The following holds

$$
\begin{aligned}
& {[f \mapsto G(\cdot, A) * f]} \\
& \quad \in \mathcal{L}_{i s}\left(\mathrm{~B}_{q, r}^{t}\left(\mathbb{R}, \mathrm{L}_{p}(0,1)\right), \mathrm{B}_{q, r}^{t+4}\left(\mathbb{R}, \mathrm{L}_{p}(0,1)\right) \cap \mathrm{B}_{q, r}^{t}\left(\mathbb{R}, \mathrm{W}_{p, \mathcal{B}}^{4}(0,1)\right)\right)
\end{aligned}
$$

Proof. The proof follows from an easy estimate for the symbol $\left(\xi^{2}+A\right)^{-1}$ since $A$ is a sectorial operator on $\mathrm{L}_{p}(0,1)$.

\subsection{A Hyperbolic Problem}

We now consider a specific instance of a hyperbolic equation which shows that our approach is capable of producing solution formulæ revealing more of the solution structure than a purely abstract (semigroup) approach. It also retains some of the transparency of the abstract approach as opposed to the fully concrete classical approach based on special functions. Consider 
the wave equation in a tube-like domain

$$
\left\{\begin{array}{cl}
u_{t t}-\triangle_{x} u-\triangle_{y} u=f, & \text { in } \mathbb{R} \times \mathbb{R}^{2} \times \Omega \ni(t, x, y), \\
u=0, & \text { in } \mathbb{R} \times \mathbb{R}^{2} \times \partial \Omega \\
u(0, \cdot)=u_{0}, & \text { in } \mathbb{R}^{2} \times \Omega \\
u_{t}(0, \cdot)=u_{1}, & \text { in } \mathbb{R}^{2} \times \Omega,
\end{array}\right.
$$

In the classical approach the role of a fundamental solution is played by the Riemann function (see for instance [p. 221][12]) which solves the system for the data $\left(f, u_{0}, u_{1}\right)=\left(0,0, \delta_{(x, y)}\right)$.

In this case we take an Hilbert space approach working in $\mathrm{L}_{2}$ and taking advantage of the functional calculus for self-adjoint operators (see for instance [Chapter 5][11]). We need to resort to this Hilbert space only functional calculus because of the well-known lack of regularization of the wave equation and the possibility of exactly characterizing Fourier multipliers in a Hilbert space setting. We go through the same steps as before. Firstly we reformulate the equation as an abstract equation in $\mathrm{L}_{2}(\Omega)$ which allows us to incorporate the boundary condition into the function space. Let $A: \operatorname{dom}(A) \subset \mathrm{L}_{2}(\Omega) \rightarrow \mathrm{L}_{2}(\Omega)$ be defined by

$$
\operatorname{dom}(A)=\left\{u \in \mathrm{H}^{2}(\Omega) \mid \gamma_{\partial \Omega} u=0\right\} \text { and } A u=-\triangle_{y} u \text { for } u \in \operatorname{dom}(A)
$$

and rewrite the above system as the following $\mathrm{L}_{2}(\Omega)$-valued wave equation on $\mathbb{R}$ for $u: \mathbb{R} \times \mathbb{R}^{2} \rightarrow \mathrm{L}_{2}(\Omega)$

$$
\left\{\begin{aligned}
u_{t t}-\triangle_{x} u+A u=f, & \text { in } \mathbb{R} \times \mathbb{R}^{2} \ni(t, x), \\
u(0, \cdot)=u_{0}, & \text { in } \mathbb{R}^{2}, \\
u_{t}(0, \cdot)=u_{1}, & \text { in } \mathbb{R}^{2}
\end{aligned}\right.
$$

A generalized Riemann function $R_{t}$ would now be a distribution satisfying this equation for $\left(f, u_{0}, u_{1}\right)=\left(0,0, \mathbf{1}_{\mathcal{L}\left(\mathrm{L}_{2}(\Omega)\right)} \delta_{x}\right)$. At this point we take a vector-valued Fourier transform in the $x$-direction to obtain the equivalent system

$$
\left\{\begin{aligned}
\hat{u}_{t t}+\left(\xi^{2}+A\right) \hat{u}=\hat{f}, & \text { in } \mathbb{R} \times \mathbb{R} \ni(t, x), \\
\hat{u}(0, \cdot)=\hat{u}_{0}, & \text { in } \mathbb{R}, \\
\hat{u}_{t}(0, \cdot)=\hat{u}_{1}, & \text { in } \mathbb{R},
\end{aligned}\right.
$$

from which the solution $\hat{R}_{t}$ can be easily computed by semigroup theory

$$
\hat{R}_{t}=\left(\xi^{2}+A\right)^{-1 / 2} \sin \left(t \sqrt{\xi^{2}+A}\right) .
$$


Here we of course use the calculus for self-adjoint operators to make sense of the formula and to lift the formula for the inverse Fourier transform for $\hat{R}_{t}$ from the scalar to the vector-valued situation. In fact, we have

$$
\hat{R}_{t}=\int_{\sigma(A)}\left(\xi^{2}+\lambda\right)^{-1 / 2} \sin \left(t \sqrt{\xi^{2}+\lambda}\right) d P(\lambda)
$$

for the spectral measure associated to $A$ (see for instance [11]).

Remark 3.1. Since we are considering the case of $\Omega$ bounded here, the spectral representation can actually be rewritten as

$$
\hat{R}_{t}=\sum_{k=1}^{\infty}\left(\xi^{2}+\lambda_{k}\right)^{-1 / 2} \sin \left(t \sqrt{\xi^{2}+\lambda_{k}}\right)\left(\cdot \mid \varphi_{k}\right)_{\mathrm{L}_{2}(\Omega)}
$$

in terms of the eigenvalues $\left(\lambda_{k}\right)_{k \in \mathbb{N}}$ and associated eigenfunctions $\left(\varphi_{k}\right)_{k \in \mathbb{N}}$ of $A$. Formula (13), however, shows that the result remains valid on more general assumptions about $\Omega$.

By continuity of the functional calculus for self-adjoint operators the Fourier transform can be taken inside the integral in (13). This gives

Theorem 3.6. The Riemann function for (12) is given by

$$
R_{t}(x, A)= \begin{cases}\frac{\cos \left(\sqrt{A}\left(t^{2}-|x|^{2}\right)^{1 / 2}\right)}{\left(t^{2}-|x|^{2}\right)^{1 / 2}}, & |x|<t \\ 0, & \text { otherwise } .\end{cases}
$$

The general solution can consequently be written as

$$
u(t, x)=\frac{d}{d t}\left(R_{t}(\cdot, A)\right) *_{x} u_{0}+R_{t}(\cdot, A) *_{x} u_{1}+\int_{0}^{t} R_{t-\tau}(\cdot, A) *_{x} f(\tau) d \tau .
$$

It should be pointed out that a pure semigroup approach would have led to the introduction of the cosine and sine families generated by the full spatial operator. In the corresponding representation formula for the solution, though equivalent, the light cone would not appear. The above formula retains both the concision and the transparency of the semigroup representation without giving up all the details of an explicit representation via classical functions. This is very important since a direct approach gives us quantitative insight into the solution whereas the functional analytical leads to a better understanding of the mapping properties of the solution operator.

In this particular case we can use the representation formula (15) to obtain the following physically interesting solution. 
Remark 3.2. Define the light cone $L$ by

$$
L:=\left\{(t, x) \in \mathbb{R} \times \mathbb{R}^{2}|| x \mid \leq t\right\}
$$

and consider the solution $u$ of (12) with initial datum

$$
\left(u_{0}, u_{1}\right)=\left(0, \delta_{x} \otimes f\right)
$$

for $f \in \mathrm{L}_{2}(\Omega)$ and where $\delta_{x}$ is the Dirac distribution supported at $x=(0,0)$. Then by (15) and (16) $u$ is given by

$$
u(t, x, \cdot)= \begin{cases}\frac{\cos \left(\sqrt{A}\left(t^{2}-|x|^{2}\right)^{1 / 2}\right)}{\left(t^{2}-|x|^{2}\right)^{1 / 2}} f, & (t, x) \in L \\ 0, & \text { otherwise }\end{cases}
$$

Since $\cos (0)=1$, it can be seen from (13) or (14) that

$$
[s \mapsto \cos (s \sqrt{A})]
$$

is strongly continuous, that is, for every $f \in \mathrm{L}_{2}(\Omega)$

$$
[s \mapsto \cos (s \sqrt{A}) f] \in \mathrm{C}\left([0, \infty), \mathrm{L}_{2}(\Omega)\right) .
$$

Notice that convergence in (14) is ensured by

$$
\left(f \mid \varphi_{k}\right)_{k \in \mathbb{N}} \in l_{2}(\mathbb{N}) .
$$

Moreover $\cos (0 \sqrt{A})=\operatorname{id}_{\mathrm{L}_{2}(\Omega)}$. We therefore obtain

$$
u(t, x) \approx \frac{f}{\left(t^{2}-|x|^{2}\right)^{1 / 2}},
$$

which shows that the singularity on the boundary of the light cone is a simple tensor product of the profile $f$ with the two dimensional scalar Riemann function for the wave equation.

\section{References}

1. H. Amann. Vector-Valued Distributions and Fourier Multipliers. http://www.math.unizh.ch/amann/books.html.

2. H. Amann. Linear and Quasilinear Parabolic Problems. Birkhäuser, Basel, 1995.

3. H. Amann. Operator-Valued Fourier Multipliers, Vector-Valued BesovSpaces, and Applications. Math. Nachr., 186:5-56, 1997.

4. G. Dore and A. Venni. On the closedness of the sum of two closed operators. Math. Z., 196:189-201, 1987.

5. I. S. Gradshteyn and I. M. Ryzhik. Tables of Integrals, Series and Products. Alan Jeffrey Editor, Academic Press, 1994. 
6. P. Guidotti. Generalized Fundamental Solutions. Accepted for publication in AAA.

7. P. Guidotti. Elliptic and parabolic problems in unbounded domains. Mathematische Nachrichten, 272:32-44, 2004.

8. A. Lunardi. Analytic Semigroups and Optimal Regularity in Parabolic Problems. Birkhäuser, Basel, 1995.

9. G. Da Prato and P. Grisvard. Maximal regularity for evolution equations by interpolation and extrapolation. J. Funct. Anal., 58:107-124, 1984.

10. L. Schwartz. Théorie des distributions à valeur vectorielles. Ann. Inst. Fourier, 7:1-141, 1957.

11. V. S. Sunder. Functional Analysis: Spectral Theory. Birkhäuser, Basel, 1991.

12. M. E. Taylor. Partial Differential Equations. Basic Theory. Springer-Verlag, New York, 1996. 